\title{
Abemaciclib: safety and effectiveness of a unique cyclin-dependent kinase inhibitor
}

Vittorio Gebbia , Maria Rosaria Valerio , Alberto Firenze \& Paolo Vigneri

To cite this article: Vittorio Gebbia , Maria Rosaria Valerio , Alberto Firenze \& Paolo Vigneri (2020): Abemaciclib: safety and effectiveness of a unique cyclin-dependent kinase inhibitor, Expert Opinion on Drug Safety, DOI: 10.1080/14740338.2020.1781814

To link to this article: https://doi.org/10.1080/14740338.2020.1781814

Accepted author version posted online: 17 Jun 2020.

Submit your article to this journal ¿

Џ Article views: 4

Q View related articles $\asymp$

View Crossmark data \lceil 
Publisher: Taylor \& Francis \& Informa UK Limited, trading as Taylor \& Francis Group

Journal: Expert Opinion on Drug Safety

DOI: $10.1080 / 14740338.2020 .1781814$

Abemaciclib: safety and effectiveness of a unique cyclin-dependent kinase inhibitor

Vittorio Gebbia ${ }^{1,2}$, Maria Rosaria Valerio ${ }^{3}$, Alberto Firenze ${ }^{4}$ and Paolo Vigneri ${ }^{5}$

${ }^{1}$ Medical Oncology Unit, La Maddalena Clinic for Cancer, Palermo, Italy

${ }^{2}$ Department of Internal Medicine "Promise," University of Palermo, Palermo, Italy

${ }^{3}$ Medical Oncology Unit, Policlinic P. Giaccone, Palermo, University of Palermo, Palermo, Italy

${ }^{4}$ Risk Management Unit, Policlinic P. Giaccone, Palermo, University of Palermo, Palermo Italy

${ }^{5}$ Medical Oncology Unit, Policlinic “G. Rodolico," University of Catania, Catania, Italy

\section{Corresponding author:}

Vittorio Gebbia,

Department of Internal Medicine "Promise," University of Palermo, Piazza delle Cliniche n. 1, 90100 Palermo, Italy

Tel.: +39-330696205

Fax: +39-0916806515

Email: vittorio.gebbia@gmail.com 


\begin{abstract}
Introduction: The discovery and the clinical availability of novel cyclin-dependent kinases 4 and 6 inhibitors have profoundly changed the therapeutic scenario of metastatic hormone receptorpositive breast carcinoma. Among these inhibitors, abemaciclib can induce potent and sustained cell cycle arrest and immune system stimulation.
\end{abstract}

Areas covered: This review summarizes the safety profile and clinical efficacy data on abemaciclib alone or in combination with aromatase inhibitors or fulvestrant in metastatic hormone receptor-positive breast carcinoma. The management of patients treated with abemaciclib is the object of this paper.

Expert opinion: As shown in phase 2 and 3 clinical trials on efficacy and tolerability, abemaciclib is a potentially convenient, safe, and effective agent for the treatment of patients with advanced hormone receptor-positive patients. Orally administered abemaciclib in combination with aromatase inhibitors or fulvestrant has the potential to allow significant improvement in survival outcomes, quality of life, response rate, and duration of response even in poor prognosis subgroups. Adequate patients' information, clinical selection, and prompt, proactive management of side effects are mandatory.

Key words: abemaciclib, breast cancer, metastases, hormonal receptors, safety 


\section{Article highlights}

- Cyclin-dependent kinase 4 and 6 inhibitor inhibitors have deeply changed the therapeutic scenario of hormone receptor positive advanced breast cancer

- Abemaciclib is an unique, potent cyclin-dependent kinase 4 and 6 inhibitor

- Clinical activity is very high with impressive results in term of patients' survival outcome

- Patients selection and drug-drug check are highly advisable before prescription

- Potentially severe side-effects are usually easily manageable but must be strictly and proactively treated and patients adequately informed 


\section{Drug summary box}

- Drug name (generic): Abemaciclib

- Phase: I-IV

- Indication: Locally advanced, recurrent or metastatic hormone sensitive breast carcinoma

- Mechanism of action: inhibition of the enzymes cyclin-dependent kinase 4 (CDK4) and cyclin-dependent kinase 6 (CDK6)

- Route of administration: orally bis in die

- Chemical structure: National Center for Biotechnology Information. PubChem Database. Abemaciclib, CID=46220502, https://pubchem.ncbi.nlm.nih.gov/compound/Abemaciclib (accessed on May 28, 2020)

- Pivotal trials: [35-40] 


\subsection{Introduction}

Nearly $75 \%$ of patients of women affected by breast cancer $(\mathrm{BC})$ have an hormone receptorpositive $(\mathrm{HR}+)$ carcinoma [1,2]. These patients receive hormone therapy $(\mathrm{HT})$ targeting the estrogen receptor-estrogen response element-regulated cell survival-signaling pathway either as a post-surgery adjuvant therapy or in the advanced/metastatic setting [3]. There are five major classes of antineoplastic agents currently used for the management of $\mathrm{HR}+\mathrm{BC}$, and they are selective estrogen receptor modulators, aromatase inhibitors (Als), selective estrogen receptor down-regulators, luteinizing hormone-releasing hormone analogs, and mTOR inhibitors. Even though all these drugs interfere with the hormone-dependent cell survival-signaling pathways in breast cancer cells, their mechanisms of action are completely different [3]. In the clinic, the presence of a primary "de novo" or the occurrence of acquired hormonal resistance limits the clinical results of Als and, more recently fulvestrant, a competitive estrogen receptor (ER) antagonist with an affinity comparable to estradiol, and the mTOR inhibitor everolimus $[4,5]$. In the last five years the therapeutic scenario of $\mathrm{HR}+$, epidermal growth factor receptor 2 negative, advanced/ metastatic breast cancer (HR+ HER2- ABC) has been profoundly changed by the clinical availability of a new class of agents which inhibit the enzymes cyclin-dependent kinase 4 (CDK4) and cyclin-dependent kinase 6 (CDK6), i.e., palbociclib, ribociclib, and abemaciclib. These agents are tolerable and able to overcome resistance to endocrine agents, as shown both in preclinical studies and in sound prospective phase III trials. To date the European Medicines Agency approved abemaciclib for "women with hormone receptor-positive, human epidermal growth factor receptor 2 negative locally advanced or metastatic breast cancer in combination with an aromatase inhibitor or fulvestrant as initial endocrine-based therapy, or in women who have received prior endocrine therapy; in pre- or perimenopausal women, the endocrine therapy should be combined with a luteinizing hormone-releasing hormone (LHRH) agonist" [6]. The FDA also included "for women and men as monotherapy in the setting of disease progression after endocrine therapy and prior chemotherapy, and as initial therapy in combination with an Al as initial endocrine therapy for postmenopausal women" [7].

The object of this paper is to review the pharmacological characteristics and clinical safety and efficacy data of abemaciclib alone or in combination with other hormonal agents.

\subsection{Preclinical data}

\subsection{Pharmacology}

In normal cellular physiology, CDK 4 and CDK 6 promote passage from the $\mathrm{G} 1$ phase to the $S$ phase of the cell cycle and are fundamental for the regulation of cell proliferation [8]. Inhibitors of cyclin-dependent kinase cyclin (CDK4/6i) currently available in the clinic fall into the class of cytostatic agents. They interact with the ATP-binding pocket of CDK4 and 6 and display a higher selectivity for CDK4 and CDK6 as compared with CDK1 and CDK2 [8]. 
Abemaciclib is a synthetic 5-(4-ethyl piperazine-1-ylmethyl)pyridin-2-yl)-(5-fluoro-4-(7-fluoro-3isopropyl-2-methyl-3H- benzoimidazol-5-yl) pyrimidine -2-yl) amine with a molecular weight of $506.606 \mathrm{~g} / \mathrm{mol}[9,10]$. Although it may act against CDK9, however, it is a highly selective CDK4/6 inhibitor 12 times more potent against CDK4 and 6 [11,12], and it has been designed for oral administration as a single-agent or in combination independently form fasting conditions $[12,13]$. Abemaciclib is more potent than other agents of the same class since it has higher inhibitory activity on CDK4 than on CDK6. Abemaciclib exerts its action even at nanomolar concentrations on CDK4/cyclinD1 (IC50=2nmol/L) and CDK6/cyclinD1 (IC50=5nmol/L) complexes [12,13] and can cross the blood-brain barrier in preclinical models [14].

\subsection{Pharmacokinetics}

In phase I studies, abemaciclib dose was progressively increased up to $275 \mathrm{mg}$ twice daily $[15,16]$. Under these conditions, abemaciclib showed a mean terminal elimination half-life ranged from 17.4 to 38.1 hours without any changes in dose-dependent clearance, a mean area under the plasma concentration-time curve over 24 hours, and at steady state reached 4280 and $5520 \mathrm{ng}-\mathrm{h} / \mathrm{mL}$ respectively for 150 and $200 \mathrm{mg}$ twice daily. At steady state, the mean maximum plasma concentration were $249 \mathrm{ng} / \mathrm{mL}$ and $298 \mathrm{ng} / \mathrm{mL}$ for $150 \mathrm{mg}$ and $200 \mathrm{mg}$ every 12 hours, respectively $[15,16]$.

After oral administration, abemaciclib display an absolute bioavailability of $45 \%$ and a plasmatic peak concentration after a median of 8 hours (range: 4.1-24.0 hours) [12,17]. Most of abemaciclib and its metabolites $(96.3 \%)$ circulate bound to plasma proteins. The primary excretion is via the fecal route $(81 \%)$ and, to a lesser extent, via renal filtration with a median plasmatic half-life of 18.3 hours. Abemaciclib is mainly metabolized in the liver by the cytochrome CYP3A4 to N-desethylabemaciclib, and a lesser extent to hydroxy derivatives (M18, M20) and another oxidative metabolite $[12,18]$. Therefore potent cytochrome inhibitors such as ketoconazole and clarithromycin and inducers such as rifampicin may have profound effects on the exposure to abemaciclib and active metabolites. Cytochrome CYP3A4 inhibitors may increase plasmatic levels of abemaciclib with the risk of severe toxicity, and conversely, CYP3A4 inducers may cause lower plasma concentrations potentially decreasing its therapeutic action.

\subsection{Mechanism of action}

\subsection{Molecular biology}

In the clinic, abemaciclib is given orally on a continuous schedule, while the other agents of the same class on an intermittent one because of hematological tolerance. In normal cellular physiology, CDK4 and CDK6 promote passage from the G1 phase to the S phase of the cell cycle and are fundamental for the regulation of cell proliferation [8]. Preclinical studies have shown that continuous inhibition of CDK4/6 by abemaciclib inhibits phosphorylation of the retinoblastoma protein, which becomes inactivated $[9,13,19]$. This effect, in turn, blocks the transition of neoplastic 
cells from the $\mathrm{G} 1$ to the $\mathrm{S}$ phase and the release of the transcription elongation factor 2 required for cell-cycle progression resulting in apoptosis or senescence [16]. This action has also been demonstrated in patients treated with neoadjuvant abemaciclib plus anastrozole. After 2 weeks of treatment, abemaciclib caused a complete cell cycle arrest, measured as $\mathrm{KI} 67$, in $68 \%$ of patients while anastrozole in only $14 \%$ of cases with a statistically significant difference $(p<0.001)[20]$. On an intent-to-treat analysis, cell cycle arrest correlated with $46 \%$ radiologic response and a $4 \%$ pathologic complete response rate.

Differently, from other CDK4/6i, abemaciclib induces extensive metabolic alterations in $\mathrm{BC}$ cells $[9,21]$, which may explain the partial absence of cross-resistance with palbociclib [16,21]. These metabolic effects correlate to an irreversible cell-cycle arrest and induction of senescence and apoptosis, which have occurred very early at doses much lower than those required for other CDK4/6i [21-23]. Abemaciclib may alter mitochondrial function, causing a time-dependent reduction in the concentration of the TCA metabolites a-ketoglutarate, fumarate, and malate, together with a minor decrease in succinate concentration. The transition from quiescence to senescence is known as geroconversion [24]. Senescence is a form of permanent growth arrest characterized by morphological changes, senescence-associated beta-galactosidase activity, presence of senescence-associated heterochromatin foci, and production of growth factors, cytokines, proteases, and other proteins and matrix-degrading molecules, collectively known as the

senescence-associated secretory phenotype.

\subsection{Immune system}

Other preclinical data suggest that abemaciclib may trigger antitumor immunity and promote of $T$ cells in the tumor microenvironment $[25,26]$. Abemaciclib in combination therapy resulted in increased cytokine signaling and adaptive immune response indicative of enhanced antigen presentation and activated T-cell phenotypes [20,21].

The interplay between CDK4/6i and the immune system is rather complicated. For instance, CDK4/6i, together with mitogen-activated protein kinase, may inhibit in vitro neoplastic cell proliferation, inducing at the same time an NK mediated retinoblastoma (RB) protein-mediated cellular senescence and activation of the immunomodulatory senescence-associated secretory phenotype leading to cellular death [27]. In patients with ABC abemaciclib plus anastrozole has been shown to determine an upregulation of inflammatory and T cell-related pathways such as PD1 signaling, IFN-gamma, and allograft rejection. At the same time, the number of tumors infiltrating lymphocytes (TIL) was not increased even if TILs were possibly activated accordingly to preclinical data $[20,28]$.

\subsection{Resistance to CDK4/6 inhibitors}


Unfortunately, patients treated with CDK4/6i sooner or later develop a progression of disease due to the emergence of cellular clones with de novo and acquired resistance [29].

Experimental preclinical investigations carried out in breast cancer cell lines, and xenografts have identified several possible mechanisms underlying resistance to CDK4/6i, such as loss of retinoblastoma susceptibility gene product function and acquired mutation in RB1, upregulation of phosphorylated PDK1and hyperactivity of cyclin A/CDK2 or cyclin E/CDK2 acquired CDK6 amplification, mutations in the phosphatidylinositol 3-kinase (PI3K) catalytic subunit [29-31]. Despite this significant amount of preclinical evidence, clinical data are almost missing [32], except PI3K mutations, which have recently targeted with alpesilib with impressive clinical results [33]. The phosphatidylinositol 3-kinase (PI3K) — protein kinase B (PKB/AKT)—mammalian target of rapamycin (mTOR) axis regulates critical physiological functions and cellular processes. This pathway has been targeted with several PI3K inhibitors with interesting results [34]. A large phase III, randomized trial total including 341 out 572 patients progressing after previous endocrine therapy and with confirmed tumor-tissue PIK3CA mutations showed that treatment with alpelisib plus fulvestrant prolonged progression-free survival (11 versus 5.7 months; HR $0.65 ; p<0.001$ ) and higher response rate (35.7\% and $16.2 \%$ ), as compared to fulvestrant plus placebo [35]. A second large, randomized, phase III studies with patients with PIK3CA mutations previously treated with an aromatase inhibitor showed a modest but significant improvement in progression-free survival with fulvestrant with taselisib versus fulvestrant plus placebo [36]. Unfortunately, in the clinic, it has not been possible to identify patients subsets who could not benefit from the addition of CDK4/6i, although patients with worse prognosis showed the most advantage [37].

\subsection{Clinical efficacy}

\subsection{Monarch-1 trial}

Efficacy of single-agent abemaciclib at the dose of $200 \mathrm{mg}$ bid was tested in a single-arm, openlabel phase II study on 132 patients with refractory HR+HER2-ABC widely pretreated with $\mathrm{HT}$ and 1-2 lines of chemotherapy [38]. Most patients (90.2\%) had visceral disease, and half had $\geq 3$ sites of metastases and a median of three lines of systemic therapy for ABC. In this reduced prognosis, cohort activity in terms of objective response was significant with an overall response rate (ORR, Recist criteria 1.1) of $19.7 \%$ (95\% Cl: $13.3-27.5)$ with no complete response and a $22.7 \%$ stabilization rate for a clinical benefit rate of $42.4 \%$. Median progression-free survival (PFS) and overall survival (OS) were 6.0 months and 17.7 months, respectively. Diarrhea, fatigue, and nausea were the most frequently reported side-effects, but toxicity-related discontinuation rate was $7.6 \%$. In this poor prognosis setting, these results were considered promising and represented the basis for further clinical development of abemaciclib.

\subsection{Monarch-2 trial}


This double-blind phase III study tested the efficacy and toxicity of abemaciclib plus fulvestrant as compared to fulvestrant alone in 669 patients with HR+/HER2-/ABC who showed disease progression during neoadjuvant or adjuvant $\mathrm{HT}$, or within 12 months form completion of adjuvant therapy, or during first-line HT for metastatic disease [39]. Pre- or perimenopausal women with ovarian suppression and postmenopausal ones were centrally randomized in a 2:1 fashion to receive fulvestrant $500 \mathrm{mg}$ every 4 weeks plus abemaciclib $150 \mathrm{mg}$ bid ( $\mathrm{n}$. 446) or placebo ( $\mathrm{n}=223$ ). At the data cut off, investigator-assessed median PFS was 16.4 and 9.3 months, respectively (HR $0.553 ; 95 \% \mathrm{Cl}, 0.449$ to $0.681 ; \mathrm{P}<0.001$ ), thus reaching statistical significance for the primary endpoint of the study. ORR, a secondary endpoint, was $48.1 \%(95 \% \mathrm{Cl}, 42.6 \%-53.6 \%)$ in the experimental arm and $21.3 \%(95 \% \mathrm{Cl}, 15.1 \%-27.6 \%)$ in the control arm respectively. An exploratory analysis of tumor shrinkage, unique to abemaciclib trials, showed that patients treated with abemaciclib have a mean change in the reduction of tumor size of $62.5 \%$ compared with $32.8 \%$ for the placebo arm [39]. Progressive disease as the best response was lower in the abemaciclib arm than in the placebo one (9.0\% versus $20.2 \%)$, and the clinical benefit rate was $72.2 \%$ and $56.1 \%$ for the abemaciclib arm and the placebo one respectively $(p=0.001)$. The 2007 paper did not report OS data because they were not mature at the time of data cutoff. The toxicity profile showed a significant excess of diarrhea in the abemaciclib arm (86.4\% v $24.7 \%)$, neutropenia $(46.0 \% \vee 4.0 \%)$, nausea $(45.1 \% \vee 22.9 \%)$, and fatigue $(39.9 \% \vee 26.9 \%)$, but clinical benefit rate was in favor of abemaciclib with excellent preservation of quality of life and safety. Sledge et al. [40] have subsequently published intent-to-treat OS data at a prespecified interim analysis with a median 46.7 months for abemaciclib plus fulvestrant and 37.3 months for placebo plus fulvestrant (HR 0.757; 95\% $0.606-0.945 ; p=0.01)$. This superiority was recorded in all stratification groups within patients with primary resistance $(\mathrm{HR}, 0.686 ; 95 \% \mathrm{Cl}, 0.451-1.043)$ and those with a visceral disease ( $\mathrm{HR}, 0.675 ; 95 \% \mathrm{Cl}, 0.511-0.891)$. The median time to second progression ( 23.1 vs. 20.6 months), the median time-to-chemotherapy (50.2 vs. 22.1 months), and median chemotherapy-free survival ( 25.5 vs. 18.2 months) were also statistically significantly superior in the abemaciclib arm than in the placebo one. The sound conclusion was that abemaciclib/fulvestrant was well tolerated and more effective than single-agent fulvestrant in $\mathrm{HR}+/ \mathrm{HER} 2-/ \mathrm{ABC}$ who progressed during previous HT. Moreover, experimental treatment was associated with a significant delay of 4.9 months in time to sustained deterioration of pain and use of analgesics as evaluated by the short form of the Brief Pain Inventory and QLQ-C30 pain item. Other symptoms such as nausea, vomiting, fatigue also were in favor of abemaciclib/ fulvestrant with the only exception of diarrhea, which significantly favored the control arm (HR 1.60; 95\% C 1.20-2.10). Global health-related quality of life functioning assessed by the EORTC QoL Core 30 and Breast Cancer Questionnaire (QLQ-BR23) assessed outcomes favored the abemaciclib arm [41].

\subsection{Monarch-3}


Goetz et al. [42] carried out a double-blind phase III study randomizing post-menopausal patients to receive abemaciclib $150 \mathrm{mg}$ bid plus anastrozole $1 \mathrm{mg} /$ day or letrozole $2,5 \mathrm{mg} /$ day $(\mathrm{n}=328)$ or placebo $(n=165)$ as the first line for metastatic disease. Median PFS, the primary study endpoint, was significantly prolonged in the abemaciclib arm (HR 0.54; 95\% Cl, 0.41-0.72; $p=0.000021$ ) with 14.7 months in the placebo arm while the median was not yet reached in the abemaciclib arm. ORR was $59 \%$ in the abemaciclib arm and $44 \%$ in the placebo arm $(p=0.004)$. In the abemaciclib arm, the most common adverse effect was diarrhea $(81.3 \%)$, even if grade 1 in nearly half of cases $(44.6 \%)$. The most frequent grade $3-4$ side-effects were neutropenia $(21.1 \% \vee 1.2 \%)$, diarrhea $(9.5 \% \vee 1.2 \%)$, and leukopenia $(7.6 \% \vee 0.6 \%)$.

Johnston et al. [43] have recently reported the final analysis of survival outcomes confirming the superiority of abemaciclib arm over the control, one being investigator-assessed median PFS significantly extended from 14,76 to 28.18 months ( $p=0.00000295 \%$; Cl 0.54;0.418-0.698). In patients with measurable disease, abemaciclib achieved a $61 \%$ ORR, which was statistically significantly higher than $45.5 \%$ reported of the placebo arm ( $p=.003$ ). Also, the median duration of response was longer in the abemaciclib arm (27.39 months) compared to the placebo arm (17.46 months). The tolerability profile was in line with previous reports. The most common grade $\geq 3$ side-effects in the abemaciclib versus placebo arms were neutropenia $(23.9 \%$ versus $1.2 \%$ ), diarrhea ( $9.5 \%$ versus $1.2 \%$ ), and leukopenia ( $8.6 \%$ versus $0.6 \%$ ).

\subsection{Prognostic factors}

Di Leo et a. [44] reported a combined subgroup analysis of 1000 patients enrolled in the Monarch2 and -3 trials to evaluate independent prognostic factors. This analysis identified prognostic factors and demonstrated that poor prognosis patients could benefit the most from the addition of abemaciclib. At univariate analysis, race, performance status, bone-only disease, visceral disease, liver metastases, progesterone receptor status, tumor grade, and the number of organs at baseline were significant. However, at multivariate analysis race, visceral disease, and the number of involved organs at baseline lost statistical association with prognosis, while performance status, bone-only disease, liver metastases, progesterone receptor status, tumor grade resulted significantly associated with prognosis indicating that these variables are necessary and the significance of one factor is independent of the others.

\subsection{Real-world and beyond-progression studies}

Since prospective clinical trials enrolled only a minority of cases because of stringent entry criteria, data collected daily form practice are essential to reassure oncologists and patients on any drug safety and efficacy in real-world patients. A retrospective analysis comprising 118 patients with HR+HER2-ABC and a median age of 66.5 years was carried out employing the Flatiron Health electronic-health record-derived database [45]. Overall, patients received abemaciclib plus fulvestrant $(59.3 \%)$, or $\mathrm{Al}(22.9 \%)$, or other treatment $(5.1 \%)$, and like the first-, second-, third- or 
further line in $28.8 \%, 21.2 \%, 20.03 \%$ and $29.7 \%$ of cases respectively. During treatment, only $6.8 \%$, and $21.2 \%$ of patients had a dose reduction or schedule change from twice to once daily. Despite the relatively small sample size and the wide heterogeneity of regimens, pretreatments, and prognostic factors, real-world median PFS was not reached after 11 months for the overall cohort, a 12-month PFS rate was $61.7 \%$. ORR was $65 \%, 37.4 \%, 35.7 \%$ and $22.3 \%$ moving from first-line to $\geq$ line. Overall, these real-world data seem in line with those achieved in controlled trials, even if they may not be fully applicable to the broader population of patients.

There is limited knowledge about the potential role of continued CDK4/6i after progression on prior CDK4/6i-based therapy. Limited experience on 12 evaluable patients treated with abemaciclib after progression on palbociclib reported five patients $(41.7 \%)$ with early progression to abemaciclib with a PFS $\leq 120$ days and three (25\%) patients responding with a PFS $>120$ days [46]. Nextgeneration sequencing of tissue samples and blood showed the presence of RB1 mutation, FGFR1 amplification, and TP53 mutation. Although most patients showed cross-resistance, a small subset of patients exposed continuously to CDKi4/6 achieved a clinical benefit, highlighting the need for additional research to evaluate potentially predictive biomarkers and drivers for the possible use of continued CDK4/6 blockade. Similar conclusions have very recently reported with the post-progression use of continuous ribociclib plus everolimus, and exemestane [47], which may be in line with data from indirect comparisons of clinical trials in CDKi4/6 naïve patients suggest that combinations of everolimus + HT may be superior to single-agent fulvestrant [48].

\subsection{Adjuvant and neo-adjuvant therapy}

The potential role of abemaciclib in adjuvant HT for HR+HER2-breast cancer is under evaluation in a multicenter, randomized, open-label phase III trial of continuous abemaciclib $150 \mathrm{mg}$ bid plus standard of care adjuvant ET [49]. Results are eagerly awaited. The neo-Monarch trial [20] explored the use of abemaciclib in the neo-adjuvant setting. This study included 223 patients randomized to receive abemaciclib $150 \mathrm{mg}$ bid150 plus anastrozole $1 \mathrm{mg} /$ day, or abemaciclib or anastrozole as single-agents. Pharmacokinetic and proliferation status, as well as mutational analysis of PIK3CA and ESR1 at baseline, were analyzed. Radiologic and caliper response rates were $46.4 \%$ and $53.6 \%$ and pathologically confirmed CR $3.7 \%$. Changes in Ki67 after exposure to abemaciclib was not associated with disease stage, baseline LN involvement, tumor grade, or tumor size. The most frequent side-effects were diarrhea (61.4\%; G3: $4.9 \%$ ), constipation (43.5\%; G3: $1.8 \%$ ), and nausea (41.7\%; G3: $2.2 \%)$. Treatment discontinuation due to AEs was low (7.6\%).

\subsection{Open questions}

\subsection{Central nervous system metastases}

Management of CNS metastases in ABC is still a major unsolved challenge [50]. Data concerning the activity of abemaciclib in this setting are scarce but intriguing since preclinical data suggest a possible synergistic activity of other CDKi4/6 with radiotherapy in brain tumors [51-53]. 
Pharmacokinetic data from a phase I study had shown that the concentration of abemaciclib in the cerebrospinal liquor approximate plasma levels [16].

Figura et al. [54] reported a retrospective analysis on the treatment of 42 brain metastases (15 patients) treated stereotactic radiotherapy for $\mathrm{HR}+$ brain within 6 months of CDK4/6 inhibitors. Five out of 15 patients received concomitant abemaciclib. Overall tolerability was excellent, and the 6and 12 -month control rate was $61 \%$ and $39 \%$, respectively, with a median OS of 36.7 months. A review of the activity of CDKI4/ on CNS metastases in patients enrolled in large randomized controlled trials have reported a substantial lack of specific data [55]. However, Tolaney et al. [56] reported an $8.7 \%$ partial response rate in a group of patients with HR+HER2-ABC in an open-label study. These results were considered interesting enough to enroll further patients. The activity of abemaciclib in patients with breast cancer, non-small cell lung cancer, or melanoma metastatic to the brain is currently under evaluation (NCT02308020) [57]. Although $16 \%$ of patients enrolled in the MonachHER trial had brain metastases at entry however it was not possible to draw significant conclusions on the activity of abemaciclib plus fulvestrant and trastuzumab in this setting even if some patients did not show progression at brain metastases [58].

\subsection{Elderly patients}

Howie et al. [59] carried out a pooled analysis that reported the efficacy and safety of any CDK4/6i plus an $\mathrm{Al}$ in 1827 elderly patients. This study confirmed efficacy in the cohort aged $\geq 75$ years with and HR $0.49(95 \% \mathrm{Cl}, 0.31$ to 0.76$)$ and a 31.1 months PFS ( $95 \% \mathrm{Cl}, 20.2$ months to not reached) for abemaciclib versus 13.7 months ( $95 \% \mathrm{Cl}, 10.9$ months to 24.9 months) for Al. However, older women experienced higher rates of grade $3-4$ toxicity $(88 \%)$ than those younger than 75 years $(73.4 \%)$, dose modifications, and a decrease from baseline in quality-of-life measures.

\subsection{Ethnicity}

The possible impact of race on efficacy and toxicity profile of any clinically available CDK4/6i is the object of a recent meta-analysis, including 2499 patients enrolled in four randomized trials [60]. Treatment with CDK4/6 inhibitors achieved a prolonged PFS compared with single-agent endocrine therapy (HR 0.56; 95\% Cl 0.50-0.62). In Asians ( $\mathrm{N}=492)$, PFS HR was $0.39(95 \% \mathrm{Cl}$ 0.29-0.51, $\mathrm{P}<0.0001)$. In non-Asians ( $\mathrm{N}=2007)$, PFS HR was 0.62 (95\% Cl 0.54-0.71, $P<0.0001)$. There was a significant treatment-by-ethnicity interaction $(P=0.002)$. Only two trials, comprising 1334 patients, reported toxicity data according to ethnicity with no soundproof of a possible link between the risk of toxicity due to CDK4/6i or endocrine monotherapy and ethnicity. The magnitude of PFS benefit is ethnicity-dependent, but there are no interethnic differences in relative treatment-related toxicities. These findings may assist in the design and interpretation of trials, inform economic analyses, and stimulate pharmacogenomic research. There was a statistically significant interaction between treatment effect on PFS and ethnicity $(P=0.002$, $)$ Interethnic difference in CYP3A4 polymorphism have been supposed to account for the differences in treatment efficacy. 


\subsection{Side-effects and management of toxicity}

\subsection{Diarrhea and other gastrointestinal toxicity}

In the MONARCH-1 trial [38], patients treated with single-agent abemaciclib experienced diarrhea in $90 \%$ of cases. Diarrhea generally ensued within one week from abemaciclib initiation and caused dosage modification in $21 \%$ of cases. In most cases, the median duration of grades 2 and 3 was 7.5 and 4.5 days, respectively. 8 In the MONARCH-2 trial [39], 73\% of patients reported grade 1 and 2 diarrhea, and $13.4 \%$ of patients also grade 3 diarrhea.9 As shown in MONARCH-1 [38], such a side-effect occurred in the first cycle, with a median duration of 6 days. Most patients (70.1\%), did not require treatment modifications 9. At the ASCO meeting in 2017, studies showed a significant decrease in diarrhea grade $3 / 4$ after cycle 4 , and 5.33 Diarrhea can weaken the patient due to loss of fluids and electrolytes. Therefore, diarrhea should be monitored carefully. Blood tests can be helpful to identify alterations in electrolyte levels, and antidiarrheal medications, such as loperamide and diphenoxylate/ atropine, should be used proactively to prevent complications.

A specially designed trial has explored the effect of diet on single-agent abemaciclib tolerability employing an e-diary in 3 different cohorts of patients under different fasting conditions defined as $\geq 1$ hour before or $\geq 2$ hours after a meal [61]. No impact of food on the incidence of prolonged grade 2 or grade 3 diarrhea and pharmacokinetic parameters was observed among patients taking single-agent abemaciclib. Moreover, the incidence and severity of diarrhea and the consequential use of loperamide were lower than expected with no grade 4 events.

Detailed patients' information on diarrhea management is mandatory since it may become, if not promptly treated, severe, and potentially life-threatening [62]. Accordingly to most guidelines, loperamide must be immediately employed at the first sign of loose stools a possibly an astringent diet and adequate oral hydration. In the case of grade 1, diarrhea supportive measures must be carried out without dose adjustments while in case of persisting or recurrent grade 2 diarrhea after resuming the same dose despite maximal supportive measures dose must be deescalated. In case of grade 3-4 diarrhea, hospitalization is mandatory, and at recovery, continue abemaciclib at the lower dose.

Abemaciclib may induce nausea/vomiting which may be treated with metoclopramide if no diarrhea or oral anti serotonin antagonists. Asymptomatic and reversible grade 3 increase of transaminases was reported in $5.8 \%$ of cases in the Monarch trials. No grade 4 was recorded. In any case dose reductions may be successfully applied as indicated for diarrhea.

\subsection{Serum creatinine impairment}

In Monarch-2 trial, serum chemistry test monitoring has shown a reversible any grade impairment in serum creatinine have been observed in $98.3 \%$ of the patients with grade $3-4$ toxicity in $1.9 \%$ of cases [39]. However, also, $78.4 \%$ of patients treated with only fulvestrant or an aromatase inhibitor 
showed an increase in serum creatinine. This increase is usually observed in the first month of therapy, tends to remain stable during exposition to abemaciclib, and is entirely reversible at discontinuation without sequelae $[16,38]$. No alterations in glomerular filtration rate as measured as iohexol clearance, cystatin C-calculated GFR or other parameters of glomerular function were found [63]. The cause of this impairment is due to the inhibition of tubular secretion of creatinine from blood to urine and metformin disposition [64]. In vitro abemaciclib and its metabolites may cause and inhibition of kidney tubular transporters involved in creatinine secretion as organic cation transporter 2, multidrug and toxin extrusion protein 1 (MATE1) and MATE2-K. No dose modifications are advisable in case of low or mild increases of serum creatinine, but caution suggests to monitor renal functions regularly. In our opinion, adequate counseling with patients and their caregiver is strongly advisable. No data exist on the use of abemaciclib in patients with basal severe kidney failure.

Abemaciclib may cause a $15-40 \%$ increases in serum creatinine levels over baseline. Compared with patients who were treated with an aromatase inhibitor or with fulvestrant alone, a creatinine serum level increase was present in $78.4 \%$ (all grades). This study assessed the in vitro and clinical inhibition of renal transporters by abemaciclib and its metabolites using metformin (a clinically relevant transporter substrate), in a clinical study that quantified glomerular filtration and iohexol clearance. In vitro, abemaciclib inhibited metformin uptake by organic cation transporter 2 , multidrug and toxin extrusion (MATE)1, and MATE2-K transporters with a half-maximal inhibitory concentration of $0.4-3.8 \mu \mathrm{M}$. In clinical trials abemaciclib significantly augments exposure to metformin but did not significantly affect glomerular filtration rate, serum neutrophil gelatinaseassociated lipocalin (NGAL), serum cystatin-C, or the urinary markers of kidney tubular injury, NGAL, and kidney injury molecule-1 [65].

\subsection{Cardiovascular side-effects and venous thromboembolism}

Potentially harmful prolongation of QTc has been reported for other CDK4/6i but not for abemaciclib [62]. Therefore checking for cardiac rhythm is advisable but not mandatory such as it is for ribociclib.

Thromboembolic events are particularly frequent in cancer patients. A specific study on all CDK4/6i recently reported no significant association between patients' characteristics and the occurrence of thromboembolism even a numerical association with a history positive for previous vascular episodes and poorly differentiated tumors [66]. The median time from start of CDK4/6i and thromboembolism was 10 months. In the Monarch-3 trial patients treated with abemaciclib plus an nonsteroidal aromatase inhibitor has a higher incidence of thromboembolic events [42]. Control for suspicion signs and symptoms of thromboembolism is advisable when prescribing and treating any patient with abemaciclib.

\subsection{Hematological toxicity}


Hematological toxicity is common during treatment with CDKi4/6 since these drugs exert their activity also on hematopoietic bone marrow inducing a reversible cell-cycle arrest reducing the proliferative activity of stem cells. Hematological toxicity is not considered a major clinical concern in patients receiving abemaciclib. However complete and differential blood cell counts need to be monitored for all patients even if hematological side-effects are usually rapidly reversible and easily managed with standard supportive care, delays, and dose modifications while continuing endocrine therapy. In the MONARCH-2 trial patients had any grade neutropenia and thrombocytopenia in $46 \%$ and $15.6 \%$ of cases respectively and received dose reductions in $42.9 \%$ and interruptions in $51.9 \%$ due to adverse events [39]. Grade 3-4 neutropenia was recorded in $26.5 \%$ of cases, and grade $3-4$ thrombocytopenia in $3.4 \%$. All grade anemia was reported in $29 \%$ of patients with grade -34 in only $7.2 \%$ of cases. Therefore blood cell counts should be recorded prior to starting abemaciclib therapy, every 2 weeks for the first 2 months, monthly for the next 2 months, and as clinically indicated in single cases. In case of grade 1 neutropenia no dose reduction is needed. In case of grade 2 neutropenia dose delay is accepted as the best way of managing toxicity, while in patients with grade 4 neutropenia both delays and dose reduction are strongly suggested. No defined suggestions are present for the management of grade 3 neutropenia since reactions depend of patients' clinical conditions.

\subsection{Drug-drug interactions}

Great attention must be paid to the concomitant use of strong CYP3A4 inhibitors when abemaciclib is prescribed [67]. Strong CYP3A4 inhibitors include antibiotics, such as clarithromycin and telithromycin, antidepressant (nefazodone), antifungal agents such as ketoconazole and itraconazole and several protease inhibitors (atazanavir, darunavir, indinavir, lopinavir, nelfinavir, ritonavir, saquinavir, tipranavir). In case of concomitant use abemaciclib dose should be reduced to $100 \mathrm{mg}$ bid, and if already employed further reduced to $50 \mathrm{mg}$ bid. Consumption of grapefruit and its juice are not advisable. Strong inhibitors may increase side effects such as nausea, vomiting, diarrhea, abdominal pain, decreased appetite, mouth sores, blood clots, hair loss, liver problems, and impaired bone marrow function resulting in low numbers of different types of blood cells. Patients may be more likely to develop anemia, bleeding problems, or infections due to low blood cell counts. On the other hand strong CYP3A4 inducers, such as rifampicin, may significantly reduce abemaciclib plasmatic concentration by $95 \%$. Therefore concomitant use of carbamazepine, phenytoin, rifampicin and St. John's wort should be taken to avoid the risk of decreased efficacy of abemaciclib. Therefore the use of a dug-checker tool is highly advised when prescribing abemaciclib.

\subsection{Expert opinion}


To date abemaciclib, unique cyclin-dependent kinase inhibitor, plays a pivotal role in the therapeutic armamentarium of inoperable, relapsed, and/or metastatic ER+ HER2- ABC. In vitro data have shown that abemaciclib is the most potent inhibitor of CDK 4 and 6 as compared to other drugs of the same class. Randomized controlled trials of abemaciclib plus letrozole or fulvestrant have shown its efficacy and safety in HR+ HER2- ABC patients with a very profound impact on progression-free and overall survival. Mature data form randomized clinical trials of abemaciclib plus fulvestrant show a significant in poor-prognosis breast cancer patients with resistant disease such as patients with progressive disease after first-line endocrine therapy or recurrent disease during adjuvant endocrine therapy or within one year from its completion. Clinical efficacy show a positive impact of abemaciclib in all patients subgroups, but clinical data also suggest to use abemaciclib in combination with endocrine therapy in breast cancer patients with visceral disease since its anti-tumour action is very potent. For instance, dramatic objective responses have reported in patients with liver metastasis, while the experience in central nervous system metastases, although very limited, seems encouraging.

Figure n. 2 shows the patient selection and treatment algorithm. Since the concomitant use of strong CYP3A4 inhibitors may significantly alter abemaciclib action its dosage should be reduced if such inhibitors are to be used. On the other hand strong CYP3A4 inducers may dramatically reduce abemaciclib plasma concentrations exposing patients to a reduction of antineoplastic activity. For these pharmacological reasons, accurate history and record of drugs use is mandatory and the routinely use of a drug-checker tools is highly advisable in clinical daily practice. Abemaciclib administration may induce hematological toxicity which however is not a dose-limiting side-effect or a reason for treatment withdrawal in any of the published experiences. On the other hand hematological toxicity is more profound and typical of other CDK4/6is. Abemaciclib is generally well tolerated by most patients and most of its side-effects are transitory or fully reversible. Its toxicity profile, therefore, allows continuous administration unique in this class of agents, which co-participate to the potent effectiveness of abemaciclib. The risk of thromboembolism is present but not more than any other hormonal agent, but it is always advisable to monitor patients for suspicious signs and symptoms. By far the most important sideeffects are diarrhea and increase in serum creatinine, the former being potentially threatening. To optimize the therapeutic index of abemaciclib careful attention should be paid in the selection of patients to be treated as well the prompt and early management of possible side effects and giving adequate information to both patients and their caregivers. A crucial point is the proactive treatment of diarrhea with loperamide. Patients should be informed about the possible increase in serum creatinine which can be ameliorated with oral hydration. Usually diarrhea reduces with time, while impairment of renal function may persist but it is fully reversible in all cases. Other sideeffects include nausea and/or vomiting which is usually successfully managed with anti-HT3 antagonists, fatigue, and rarely alopecia. 
In our experience, abemaciclib should be considered in patients with hormone-refractory $A B C$ in combination with fulvestrant, even if results in hormone-sensitive patients largely justify its choice in combination with aromatase inhibitors as the first-line option.

\section{Funding}

This paper was not funded.

\section{Declaration of interests}

V. Gebbia, M.R. Valerio and P. Vigneri have received honoraria for the participation of an advisory board for Pfizer, Novartis, and Eli Lilly. The authors have no other relevant affiliations or financial involvement with any organization or entity with a financial interest in or financial conflict with the subject matter or materials discussed in the manuscript. This includes employment, consultancies, honoraria, stock ownership or options, expert testimony, grants or patents received or pending, or royalties.

\section{Reviewer disclosures}

A reviewer on this manuscript has disclosed that they are an advisory board member or have received speakers bureau, honoraria and travel support from Pfizer, Lilly, Novartis, Roche and AstraZeneca. All other peer reviewers on this manuscript have no relevant financial relationships or otherwise to disclose. 


\section{References}

Papers of special note have been highlighted as:

${ }^{*}$ of interest

** of considerable interest

[1] Nadji M, Gomez-Fernandez C, Ganjei-Azar P, et al. Immunohistochemistry of estrogen and progesterone receptors reconsidered: experience with 5,993 breast cancers. Am J Clin Pathol. 2005;123:21-27.

[2] Cancer Genome Atlas Network. Comprehensive molecular portraits of human breast tumours. Nature. 2012;490:61-70.

[3] Hormone Therapy for Breast Cancer | American Cancer Society [Internet]. [cited 2020 May 29]. Available from: https://www.cancer.org/cancer/breast-cancer/treatment/hormone-therapyfor-breast-cancer.html.

[4] Piccart M, Hortobagyi GN, Campone M, et al. Everolimus plus exemestane for hormonereceptor-positive, human epidermal growth factor receptor-2-negative advanced breast cancer: overall survival results from BOLERO-2†. Ann Oncol Off J Eur Soc Med Oncol. 2014;25:2357-2362.

[5] Robertson JFR, Jiang Z, Di Leo A, et al. A meta-analysis of clinical benefit rates for fulvestrant $500 \mathrm{mg}$ vs. alternative endocrine therapies for hormone receptor-positive advanced breast cancer. Breast Cancer Tokyo Jpn. 2019;26:703-711.

[6] verzenios-epar-product-information_en.pdf [Internet]. [cited 2020 May 29]. Available from: https://www.ema.europa.eu/en/documents/product-information/verzenios-epar-productinformation_en.pdf.

[7] VERZENIO - HIGHLIGHTS OF PRESCRIBING INFORMATION.pdf [Internet]. [cited 2020 May 29]. Available from: https://www.accessdata.fda.gov/drugsatfda_docs/label/2018/208855s000lbl.pdf.

[8] Vermeulen K, Van Bockstaele DR, Berneman ZN. The cell cycle: a review of regulation, deregulation and therapeutic targets in cancer. Cell Prolif. 2003;36:131-149.

[9] Torres-Guzmán R, Calsina B, Hermoso A, et al. Preclinical characterization of abemaciclib in hormone receptor positive breast cancer. Oncotarget. 2017;8:69493-69507.

[10] Abemaciclib Monograph for Professionals [Internet]. Drugs.com. [cited 2020 May 29].

Available from: https://www.drugs.com/monograph/abemaciclib.html.

[11] Lallena MJ, Boehnke K, Torres R, et al. Abstract 3101: In-vitro characterization of Abemaciclib pharmacology in ER+ breast cancer cell lines. Mol Cell Biol [Internet]. American Association for Cancer Research; 2015 [cited 2020 May 29]. p. 3101-3101. Available from: http://cancerres.aacrjournals.org/lookup/doi/10.1158/1538-7445.AM2015-3101.

[12] O'Leary B, Finn RS, Turner NC. Treating cancer with selective CDK4/6 inhibitors. Nat Rev Clin Oncol. 2016;13:417-430. 
[13] Gelbert LM, Cai S, Lin X, et al. Preclinical characterization of the CDK4/6 inhibitor LY2835219: in-vivo cell cycle-dependent/independent anti-tumor activities alone/in combination with gemcitabine. Invest New Drugs. 2014;32:825-837.

[14] Sanchez-Martinez C, Gelbert LM, Shannon H, et al. Abstract B234: LY2835219, a potent oral inhibitor of the cyclin-dependent kinases 4 and $6(C D K 4 / 6)$ that crosses the blood-brain barrier and demonstrates in vivo activity against intracranial human brain tumor xenografts. Mol Cancer Ther. 2011;10:B234-B234.

[15] Shapiro G, Rosen LS, Tolcher AW, et al. A first-in-human phase I study of the CDK4/6 inhibitor, LY2835219, for patients with advanced cancer. J Clin Oncol. 2013;31:2500-2500.

[16] Patnaik A, Rosen LS, Tolaney SM, et al. Efficacy and Safety of Abemaciclib, an Inhibitor of CDK4 and CDK6, for Patients with Breast Cancer, Non-Small Cell Lung Cancer, and Other Solid Tumors. Cancer Discov. 2016;6:740-753.

[17] Robert M, Frenel J-S, Bourbouloux E, et al. Pharmacokinetic drug evaluation of abemaciclib for advanced breast cancer. Expert Opin Drug Metab Toxicol. 2019;15:85-91.

[18] Posada MM, Morse BL, Turner PK, et al. Predicting Clinical Effects of CYP3A4 Modulators on Abemaciclib and Active Metabolites Exposure Using Physiologically Based Pharmacokinetic Modeling. J Clin Pharmacol. 2020;

[19] Goodrich DW, Wang NP, Qian YW, et al. The retinoblastoma gene product regulates progression through the G1 phase of the cell cycle. Cell. 1991;67:293-302.

[20] *Hurvitz SA, Martin M, Press MF, et al. Potent Cell-Cycle Inhibition and Upregulation of Immune Response with Abemaciclib and Anastrozole in neoMONARCH, Phase II Neoadjuvant Study in HR+/HER2- Breast Cancer. Clin Cancer Res Off J Am Assoc Cancer Res. 2020;26:566-580.

This study gives informations on the activity of abemaciclib in the neoadjuvant setting

[21] Bonelli M, La Monica S, Fumarola C, et al. Multiple effects of CDK4/6 inhibition in cancer: From cell cycle arrest to immunomodulation. Biochem Pharmacol. 2019;170:113676.

[22] Zou X, Ray D, Aziyu A, et al. Cdk4 disruption renders primary mouse cells resistant to oncogenic transformation, leading to Arf/p53-independent senescence. Genes Dev. 2002;16:2923-2934.

[23] Baughn LB, Di Liberto M, Wu K, et al. A novel orally active small molecule potently induces G1 arrest in primary myeloma cells and prevents tumor growth by specific inhibition of cyclindependent kinase 4/6. Cancer Res. 2006;66:7661-7667.

[24] Blagosklonny MV. Geroconversion: irreversible step to cellular senescence. Cell Cycle Georget Tex. 2014;13:3628-3635.

[25] Schaer DA, Beckmann RP, Dempsey JA, et al. The CDK4/6 Inhibitor Abemaciclib Induces a T Cell Inflamed Tumor Microenvironment and Enhances the Efficacy of PD-L1 Checkpoint Blockade. Cell Rep. 2018;22:2978-2994. 
[26] Goel S, DeCristo MJ, Watt AC, et al. CDK4/6 inhibition triggers anti-tumour immunity. Nature. 2017;548:471-475.

[27] Ruscetti M, Leibold J, Bott MJ, et al. NK cell-mediated cytotoxicity contributes to tumor control by a cytostatic drug combination. Science. 2018;362:1416-1422.

[28] Teh JLF, Aplin AE. Arrested Developments: CDK4/6 Inhibitor Resistance and Alterations in the Tumor Immune Microenvironment. Clin Cancer Res Off J Am Assoc Cancer Res. 2019;25:921-927.

[29] Pandey K, An H-J, Kim SK, et al. Molecular mechanisms of resistance to CDK4/6 inhibitors in breast cancer: A review. Int J Cancer. 2019;145:1179-1188.

[30] Niu Y, Xu J, Sun T. Cyclin-Dependent Kinases 4/6 Inhibitors in Breast Cancer: Current Status, Resistance, and Combination Strategies. J Cancer. 2019;10:5504-5517.

[31] McCartney A, Migliaccio I, Bonechi M, et al. Mechanisms of Resistance to CDK4/6 Inhibitors: Potential Implications and Biomarkers for Clinical Practice. Front Oncol. 2019;9:666.

[32] Guarducci C, Bonechi M, Boccalini G, et al. Mechanisms of Resistance to CDK4/6 Inhibitors in Breast Cancer and Potential Biomarkers of Response. Breast Care Basel Switz. 2017;12:304-308.

[33] Rozeboom B, Dey N, De P. ER+ metastatic breast cancer: past, present, and a prescription for an apoptosis-targeted future. Am J Cancer Res. 2019;9:2821-2831.

[34] Verret B, Cortes J, Bachelot T, et al. Efficacy of PI3K inhibitors in advanced breast cancer. Ann Oncol Off J Eur Soc Med Oncol. 2019;30:x12-x20.

[35] André F, Ciruelos E, Rubovszky G, et al. Alpelisib for PIK3CA-Mutated, Hormone ReceptorPositive Advanced Breast Cancer. N Engl J Med. 2019;380:1929-1940.

[36] Baselga J, Dent SF, Cortés J, et al. Phase III study of taselisib (GDC-0032) + fulvestrant (FULV) v FULV in patients (pts) with estrogen receptor (ER)-positive, PIK3CA-mutant (MUT), locally advanced or metastatic breast cancer (MBC): Primary analysis from SANDPIPER. J Clin Oncol. 2018;36:LBA1006-LBA1006.

[37] *Giordano SH, Elias AD, Gradishar WJ. NCCN Guidelines Updates: Breast Cancer. J Natl Compr Cancer Netw JNCCN. 2018;16:605-610.

This reference reported updated guidelines for the treatment of breast cancer

[38] Dickler MN, Tolaney SM, Rugo HS, et al. MONARCH 1, A Phase II Study of Abemaciclib, a CDK4 and CDK6 Inhibitor, as a Single Agent, in Patients with Refractory HR+/HER2Metastatic Breast Cancer. Clin Cancer Res Off J Am Assoc Cancer Res. 2017;23:52185224.

[39] Sledge GW, Toi M, Neven P, et al. MONARCH 2: Abemaciclib in Combination With Fulvestrant in Women With HR+/HER2- Advanced Breast Cancer Who Had Progressed While Receiving Endocrine Therapy. J Clin Oncol Off J Am Soc Clin Oncol. 2017;35:28752884. 
[40] *Sledge GW, Toi M, Neven P, et al. The Effect of Abemaciclib Plus Fulvestrant on Overall Survival in Hormone Receptor-Positive, ERBB2-Negative Breast Cancer That Progressed on Endocrine Therapy-MONARCH 2: A Randomized Clinical Trial. JAMA Oncol. 2019;

This study demonstrates superiority of abemaciclib plus fulvestrant in hormone-resistant patients with metastatic breast cancer.

[41] Kaufman PA, Toi M, Neven P, et al. Health-Related Quality of Life in MONARCH 2: Abemaciclib plus Fulvestrant in Hormone Receptor-Positive, HER2-Negative Advanced Breast Cancer After Endocrine Therapy. The Oncologist. 2020;25:e243-e251.

[42] Goetz MP, Toi M, Campone M, et al. MONARCH 3: Abemaciclib As Initial Therapy for Advanced Breast Cancer. J Clin Oncol Off J Am Soc Clin Oncol. 2017;35:3638-3646.

[43] Johnston S, Martin M, Di Leo A, et al. MONARCH 3 final PFS: a randomized study of abemaciclib as initial therapy for advanced breast cancer. NPJ Breast Cancer. 2019;5:5.

[44] Di Leo A, O'Shaughnessy J, Sledge GW, et al. Prognostic characteristics in hormone receptor-positive advanced breast cancer and characterization of abemaciclib efficacy. NPJ Breast Cancer. 2018;4:41.

[45] Carter GC, Sheffield KM, Gossai A, et al. Abstract P2-08-12: Initial real world treatment patterns and outcomes of Abemaciclib for the treatment of HR+,HER2- metastatic breast cancer. Cancer Res. 2020;80:P2-P2-08-12.

[46] Wander SA, Spring LM, Stein CR, et al. Abstract P6-18-39: Abemaciclib after prior palbociclib exposure in patients with metastatic hormone-receptor positive $(\mathrm{HR}+) / \mathrm{HER} 2-$ breast cancer. Cancer Res. 2019;79:P6-P6-18-39.

[47] Triplet therapy (continuous ribociclib, everolimus, exemestane) in HR+/HER2- advanced breast cancer postprogression on a CDK4/6 inhibitor (TRINITI-1): Efficacy, safety, and biomarker results. | Journal of Clinical Oncology [Internet]. [cited 2020 May 29]. Available from: https://ascopubs.org/doi/10.1200/JCO.2019.37.15_suppl.1016.

[48] Rossi L, McCartney A, Risi E, et al. Managing advanced HR-positive, HER2-negative breast cancer with CDK4/6 inhibitors in post-menopausal patients: is there a best sequence? Ther Adv Med Oncol. 2018;10:1758835918815591.

[49] Rastogi P, Toi M, Harbeck N, et al. Abstract OT3-05-05: MonarchE: A randomized, openlabel, phase 3 study of abemaciclib combined with standard adjuvant endocrine therapy versus standard adjuvant endocrine therapy alone in patients with high risk, node positive, early stage, HR+, HER2- breast cancer. Cancer Res. 2018;78:OT3-OT3-05-05.

[50] O'Sullivan CC, Davarpanah NN, Abraham J, et al. Current challenges in the management of breast cancer brain metastases. Semin Oncol. 2017;44:85-100.

[51] Whittaker S, Madani D, Joshi S, et al. Combination of palbociclib and radiotherapy for glioblastoma. Cell Death Discov. 2017;3:17033. 
[52] Hashizume R, Zhang A, Mueller S, et al. Inhibition of DNA damage repair by the CDK4/6 inhibitor palbociclib delays irradiated intracranial atypical teratoid rhabdoid tumor and glioblastoma xenograft regrowth. Neuro-Oncol. 2016;18:1519-1528.

[53] Naz S, Cook JA, Mitchell JB. Abemaciclib: A multi-functional radiation modifier. Oncotarget. 2019;10:1230-1232.

[54] Figura NB, Potluri TK, Mohammadi $\mathrm{H}$, et al. CDK 4/6 inhibitors and stereotactic radiation in the management of hormone receptor positive breast cancer brain metastases. J Neurooncol. 2019;144:583-589.

[55] Nguyen LV, Searle K, Jerzak KJ. Central nervous system-specific efficacy of CDK4/6 inhibitors in randomized controlled trials for metastatic breast cancer. Oncotarget. 2019;10:6317-6322.

[56] Tolaney SM, Lin NU, Thornton D, et al. Abemaciclib for the treatment of brain metastases (BM) secondary to hormone receptor positive $(\mathrm{HR}+)$, HER2 negative breast cancer. J Clin Oncol. 2017;35:1019-1019.

[57] Shah N, Mohammad AS, Saralkar P, et al. Investigational chemotherapy and novel pharmacokinetic mechanisms for the treatment of breast cancer brain metastases. Pharmacol Res. 2018;132:47-68.

[58] Tolaney SM, Wardley AM, Zambelli S, et al. Abemaciclib plus trastuzumab with or without fulvestrant versus trastuzumab plus standard-of-care chemotherapy in women with hormone receptor-positive, HER2-positive advanced breast cancer (monarcHER): a randomised, openlabel, phase 2 trial. Lancet Oncol. 2020;

[59] Howie LJ, Singh H, Bloomquist E, et al. Outcomes of Older Women With Hormone ReceptorPositive, Human Epidermal Growth Factor Receptor-Negative Metastatic Breast Cancer Treated With a CDK4/6 Inhibitor and an Aromatase Inhibitor: An FDA Pooled Analysis. J Clin Oncol Off J Am Soc Clin Oncol. 2019;37:3475-3483.

[60] Lee KWC, Lord S, Finn RS, et al. The impact of ethnicity on efficacy and toxicity of cyclin D kinase 4/6 inhibitors in advanced breast cancer: a meta-analysis. Breast Cancer Res Treat. 2019;174:271-278.

[61] Abstract P5-14-05: The impact of food on tolerability of abemaciclib in patients with previously treated hormone receptor-positive, HER2-negative, metastatic breast cancer: An open-label, randomized phase 2 study | Cancer Research [Internet]. [cited 2020 May 29]. Available from: https://cancerres.aacrjournals.org/content/80/4_Supplement/P5-14-05.

[62] Thill M, Schmidt M. Management of adverse events during cyclin-dependent kinase 4/6 (CDK4/6) inhibitor-based treatment in breast cancer. Ther Adv Med Oncol. 2018;10:1758835918793326.

[63] Krutzén E, Bäck SE, Nilsson-Ehle I, et al. Plasma clearance of a new contrast agent, iohexol: a method for the assessment of glomerular filtration rate. J Lab Clin Med. 1984;104:955-961. 
[64] Idkaidek N, Arafat T. Metformin IR versus XR Pharmacokinetics in Humans. J Bioequivalence Bioavailab [Internet]. 2011 [cited 2020 May 29];03. Available from:

https://www.omicsonline.org/metformin-ir-versus-xr-pharmacokinetics-in-humansjbb. 1000092. php?aid=2319.

[65] Chappell JC, Turner PK, Pak YA, et al. Abemaciclib Inhibits Renal Tubular Secretion Without Changing Glomerular Filtration Rate. Clin Pharmacol Ther. 2019;105:1187-1195.

[66] Gervaso L, Montero AJ, Jia X, et al. Venous thromboembolism in breast cancer patients receiving cyclin-dependent kinase inhibitors. J Thromb Haemost JTH. 2020;18:162-168.

[67] Ettl J. Management of Adverse Events Due to Cyclin-Dependent Kinase 4/6 Inhibitors. Breast Care Basel Switz. 2019;14:86-92. 
Figure n. 1. Patient selection and treatment algorithm

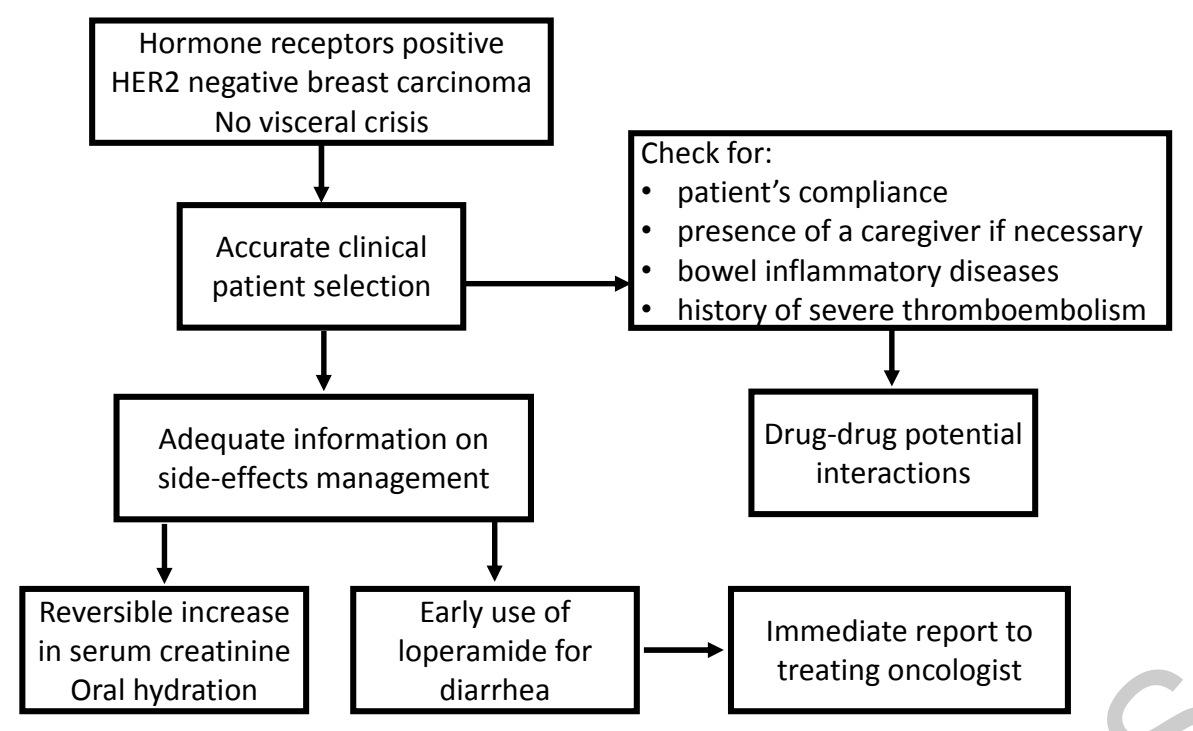

